\title{
A Case-Control Study to Investigate the Serotypes of S. suis Isolates by Multiplex PCR in Nursery Pigs in Ontario, Canada
}

\author{
Leann C. Denich ${ }^{1, *}$, Abdolvahab Farzan ${ }^{1}{ }^{\circledR}$, Robert Friendship ${ }^{1}$, Emily Arndt ${ }^{1}$, \\ Marcelo Gottschalk ${ }^{2}$ and Zvonimir Poljak ${ }^{1}$ \\ 1 Department of Population Medicine, University of Guelph, 50 Stone Rd E, Guelph, ON N1G 2W1, Canada; \\ afarzan@uoguelph.ca (A.F.); rfriends@ovc.uoguelph.ca (R.F.); arndte@uoguelph.ca (E.A.); \\ zpoljak@uoguelph.ca (Z.P.) \\ 2 The Research Group on Infectious Diseases in Production Animals, University of Montreal, 3200 Sicotte, \\ Saint-Hyacinthe, Montréal, QC J2S 2M2, Canada; marcelo.gottschalk@umontreal.ca \\ * Correspondence: ldenich@uoguelph.ca
}

Received: 1 December 2019; Accepted: 2 January 2020; Published: 5 January 2020

check for updates

\begin{abstract}
Streptococcus suis naturally inhabits the tonsils and nasal cavities of pigs. Some strains can cause systemic infection, leading to a wide range of diseases. A case-control study was conducted to (i) examine serotypes isolated from systemic sites (blood/meninges/spleen) in cases, (ii) determine whether serotypes in systemic sites were found in upper respiratory sites (tonsil/nasal cavity) of the same cases, and (iii) determine the serotypes in upper respiratory sites of case and farm and pen- matched controls. In total, 606 samples from 128 pigs were cultured for $S$. suis. The isolates were examined for presence of $g d h$ and $r e c N$ genes by polymerase chain reaction (PCR) and were identified as $S$. suis if both genes were present. The $S$. suis isolates were then serotyped using a two step-multiplex PCR. Serotypes $9(n=9),(2,1 / 2)(n=7)$ and untypable isolates $(n=7)$ were most commonly found in systemic sites. Detection of serotypes $9(p=0.03)$ in upper respiratory sites were positively associated with their detection in systemic sites of cases, while a trend was seen with serotype $(2,1 / 2)(p=0.07)$. Last, no association between serotypes recovered from upper respiratory sites of cases and controls could be detected. Untypable isolates were detected in high frequency, which warrants further investigation. This study confirms that a variety of serotypes can be found in commercial swine production and shows a difference in serotypes recovered from systemic sites in pigs with clinical signs of $S$. suis infections.
\end{abstract}

Keywords: swine; Streptococcus suis; serotypes; systemic and non-systemic sites

\section{Background}

Streptococcus suis infections have become a major problem in the swine industry worldwide [1]. This bacterium is a Gram-positive facultative anaerobe that naturally inhabits the tonsils and nasal cavities of pigs, with most pigs remaining healthy carriers. Outbreaks tend to be sporadic and generally involve only a small number of at-risk pigs. Typically, the age group at greatest risk of developing clinical signs of infection are four- to eight-week-old piglets, and systemic infection in these pigs can result in a wide range of disease conditions [2]. In addition, S. suis is potentially zoonotic, with people who work closely with infected pigs or pork-derived products being at greatest risk in western countries [3]. Worldwide, there has been over 1000 S. suis human cases and over 100 deaths since 1968, with the most cases occurring in Vietnam and Thailand through the ingestion of contaminated raw pork products [4,5]. 
Twenty-nine known serotypes have previously been identified based on the capsular polysaccharide (cps) [4]. Most S. suis isolates recovered from diseased pigs belong to serotypes 1 through 9, 1/2 and 14, though the distribution may differ depending on geographical location $[5,6]$. In Canada, the most frequent serotypes recorded in diseased pigs (in decreasing order) are serotypes, namely 2, 3, 1/2, 8, 4, and $7[4,7,8]$. There are also untypable strains, which may be novel serotypes or mutants of known serotypes [1].

The most commonly recognized clinical expression of $S$. suis infection include neurological clinical signs, including ataxia, incoordination, tremor/shaking, paddling, opisthotonos, paralysis, convulsions, nystagmus, and often sudden death [6]. Disease tends to have a low incidence $(<5 \%)$ on most farms. Factors related to host (immunity, upper respiratory diseases, microbiome, and genetics), environment (farm management, stocking density, ventilation, and temperature) and bacteria (virulence associated factors) likely play a role in the development of clinical illness. However, there are knowledge gaps with respect to why some animals become systemically ill, while most remain healthy, and how the bacterium spreads among pigs in a specific population. Both uncertainties add additional challenges when attempting to determine what triggers some pigs to become sick. A pig-level, farmand pen-matched case-control study was conducted with the overarching goal to understand factors contributing to $S$. suis disease status in nursery pigs.

The objectives of this study were to: (i) examine S. suis serotypes isolated from systemic infections in sick pigs in Ontario, Canada, (ii) investigate whether serotypes found in systemic sites of sick pigs were also found in the upper respiratory sites of the same pigs, and (iii) compare the serotypes found in upper respiratory sites of sick and healthy pigs.

\section{Results}

\subsection{Streptococcus suis Serotypes Recovered from All Samples}

In total, 128 pigs (64 healthy pigs and 64 pigs with clinical signs of $S$. suis infection) on 12 farms were sampled. A total of 606 samples were collected, of which 359 were from clinically ill (case) pigs and 247 were from healthy (control) pigs. In total, 310 S. suis isolates ( 215 from cases and 95 from controls) were recovered. Streptococcus suis was isolated from at least one sample in 114 pigs (60 cases and 54 controls). At the pig level, S. suis was recovered most frequently from the tonsillar swabs in $69.4 \%$ of cases and $58.7 \%$ of controls (Table 1 ). The multiplex PCR was able to determine the serotypes of 178 typable isolates ( 122 from cases and 56 from controls), while 132 isolates ( 74 from cases and 58 from controls) remained untypable.

Table 1. The number of samples collected and tested positive for S. suis from case (sick with S. suis clinical signs) and control (healthy) animals at the pig level.

\begin{tabular}{ccccccc}
\hline & \multicolumn{7}{c}{ Pig Level } \\
\cline { 2 - 7 } Sample-Type & \multicolumn{5}{c}{ Cases } & Controls \\
\cline { 2 - 7 } & $\begin{array}{c}\text { Pigs } \\
\text { Sampled (n) }\end{array}$ & $\begin{array}{c}\text { Pigs Tested } \\
\text { Positive (n) }\end{array}$ & $\begin{array}{c}\text { \% Pigs } \\
\text { Positive }\end{array}$ & $\begin{array}{c}\text { Pigs } \\
\text { Sampled (n) }\end{array}$ & $\begin{array}{c}\text { Pigs Tested } \\
\text { Positive (n) }\end{array}$ & $\begin{array}{c}\text { \% Pigs } \\
\text { Positive }\end{array}$ \\
\hline Blood & 62 & 9 & 14.5 & 62 & 0 & 0 \\
Meningeal swabs & 64 & 25 & 39.1 & n/a & n/a & n/a \\
Spleen tissue & 48 & 5 & 10.4 & n/a & n/a & n/a \\
Tonsillar swabs & 62 & 43 & 69.4 & 63 & 37 & 58.7 \\
Nasal cavity swabs & 60 & 27 & 45.0 & 64 & 17 & 26.6 \\
Rectal swabs & 63 & 22 & 34.9 & 58 & 10 & 17.2 \\
Total & 64 & 60 & 93.7 & 64 & 54 & 84.4 \\
\hline
\end{tabular}

Seventeen known serotypes and untypable isolates were recovered from upper respiratory (tonsil/nasal cavity) sites in 47 of 64 case and in 38 of 64 control animals (Figure 1), and 10 known serotypes and untypable isolates were recovered from rectal sites in 22 of 64 case and in 10 of 64 control animals (Figure 2). Serotypes recovered most frequently from case animals in upper respiratory sites 
(tonsil/nasal cavity) included $(2,1 / 2)(n=8), 15(n=6), 29(n=6), 16(n=5)$, and $30(n=5)$, as well as untypable isolates $(n=30)$, while the most frequent serotypes recovered from the rectum of case animals included $9(n=3)$ and $30(n=3)$, as well as untypable isolates $(n=15)$. In control animals, the most frequent serotypes recovered in upper respiratory sites (tonsil/nasal cavity) included $29(\mathrm{n}=7), 7$ $(n=4), 16(n=4), 18(n=4)$, and $9(n=3)$, as well as untypable isolates $(n=24)$, while serotypes most frequently recovered from the rectum of control animals included serotypes $9(n=3), 28(n=2)$, and untypable isolates $(n=3)$.

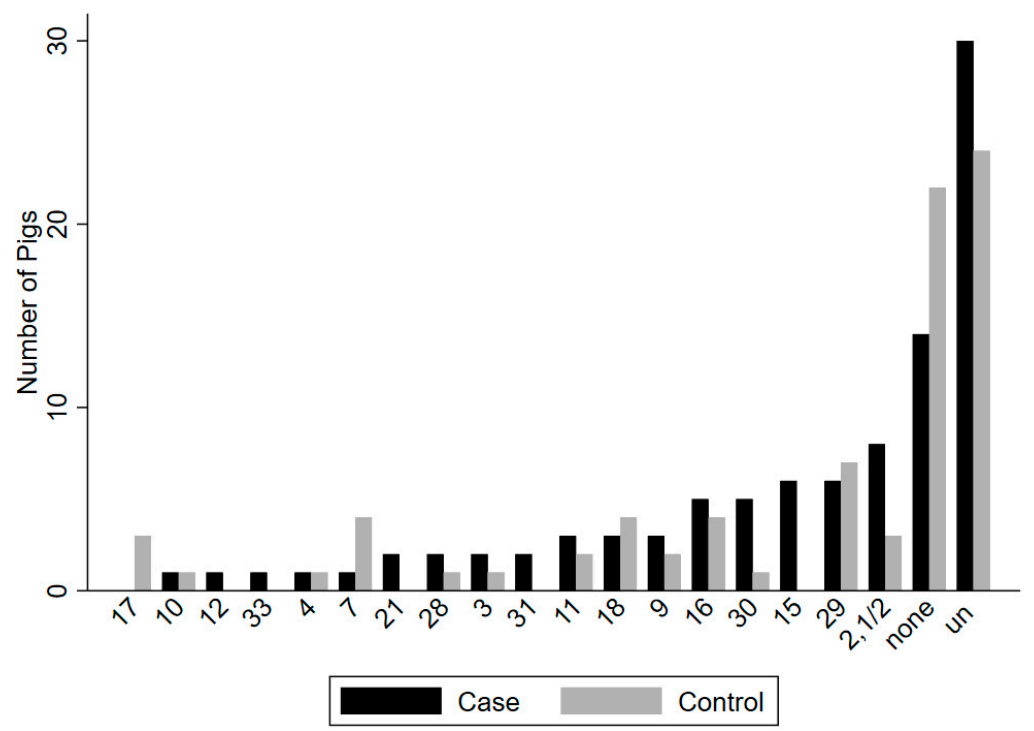

Figure 1. Comparison of the number of Streptococcus suis serotypes detected in upper respiratory (tonsil/nasal cavity) sites in 47 of 64 case animals and in 38 of 64 control animals on 12 Ontario farms. The most frequent serotypes in case animals included $(2,1 / 2)(n=8), 15(n=6), 16(n=5), 29(n=6)$, and $30(n=5)$, along with untypable (un) isolates $(n=30)$. The most frequent serotypes in control animals included $29(n=7), 9(n=3), 7(n=4), 16(n=4)$, and $18(n=4)$, along with untypable isolates $(n=24)$.

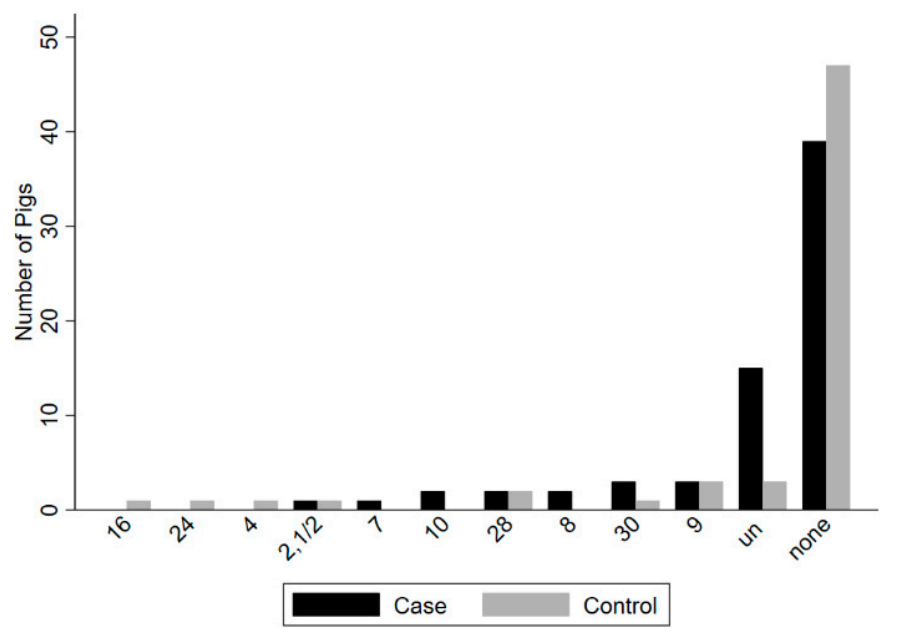

Figure 2. Comparison of the number of Streptococcus suis serotypes detected from rectal swabs in 25 of 64 clinical case animals and in nine of 64 control animals on 12 Ontario farms. The most frequent serotypes in case animals included $9(n=3), 30(n=3)$ and untypable (un) isolates $(n=15)$. The most frequent serotypes in control animals included serotype $9(n=3)$ and $28(n=2)$, as well as untypable isolates $(n=3)$. 
When considering all sample types, a single serotype was found in 12 case animals and 18 control animals, while two or more serotypes were recovered in 47 case animals and 22 control animals. With respect to the population of clinical cases, tonsillar swabs had the greatest number of different serotypes recovered $(\mathrm{n}=13)$, followed by nasal cavity swabs $(\mathrm{n}=12)$ and meningeal swabs $(\mathrm{n}=11)$. Similarly, in the population of controls, tonsillar swabs also had the greatest number of different serotypes recovered $(n=12)$, followed by nasal cavity swabs $(n=8)$ and rectal swabs $(n=7)$.

The number of different serotypes found in 64 sampled clinical case pigs recovered on 12 farms can be seen in Figure 3 and the different serotypes found on each farm can be seen in Table 2. The number of serotypes detected at the level of the individual farm were between one and 15, with an average of seven.

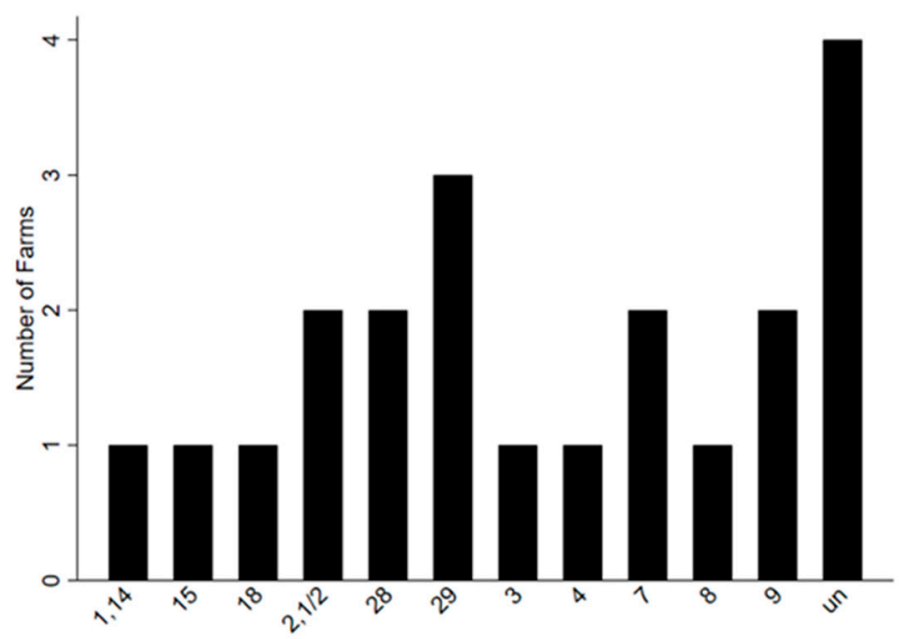

Figure 3. Number of Streptococcus suis serotypes detected on 12 farms in systemic (blood, meninges, spleen) sites of 28 confirmed cases with clinical signs of $S$. suis infections. The serotypes identified from most farms were $29(n=3), 7(n=2), 9(n=2), 28(n=2)$, and $(2,1 / 2)(n=2)$, as well as untypable (un) isolates $(n=4)$. 
Table 2. Streptococcus suis serotypes found in case and control animals on each study farm based off all sampling sites.

\begin{tabular}{|c|c|c|c|c|c|c|c|c|c|c|c|c|c|c|c|c|c|c|c|c|c|c|c|c|c|}
\hline Farm & Health Status & 1,14 & $2,1 / 2$ & 3 & 4 & 5 & 7 & 8 & 9 & 10 & 11 & 12 & 15 & 16 & 17 & 18 & 21 & 24 & 28 & 29 & 30 & 31 & 33 & 34 & un \\
\hline \multirow[t]{2}{*}{1} & Case & & & & & & & & + & & & & + & + & & & & & & & & + & & & + \\
\hline & Control & & & & & & & & & & & & + & + & & & & & & + & & & & & + \\
\hline \multirow[t]{2}{*}{2} & Case & & & & & & & & & & & & & & & & & & & & & & & & \\
\hline & Control & & & & & & & & & & & & & & & & & & & & & + & & & \\
\hline \multirow[t]{2}{*}{3} & Case & & & & & & & & & & & & + & & & & + & & & + & & & & & + \\
\hline & Control & & & & & & + & & & & & & & & + & & & & & + & & & & & + \\
\hline \multirow[t]{2}{*}{4} & Case & & + & + & & & + & + & & & & & + & & & & & & & + & + & + & & & + \\
\hline & Control & & & & & & + & & & & & & & + & + & & & & & + & & + & & + & + \\
\hline \multirow[t]{2}{*}{6} & Case & & & & & & & & + & & + & & & + & & & & & & & + & & & & + \\
\hline & Control & & & & & & & & & & & & & + & & & & & & + & + & & & & + \\
\hline \multirow[t]{2}{*}{7} & Case & & + & & + & + & + & + & & + & + & & + & + & & + & & & + & + & + & + & & & + \\
\hline & Control & & + & & & & + & & & & + & & & & & + & & & & + & + & & & & + \\
\hline \multirow[t]{2}{*}{8} & Case & & + & & & & & & + & & & & & & & & & & + & & & & & & + \\
\hline & Control & & & & + & & & + & & & & & & & & & + & & & & & & & & + \\
\hline \multirow[t]{2}{*}{9} & Case & & & & & & & & & & & & & & & & & & & & & & & & + \\
\hline & Control & & & & & & & & & & & & & & & & & + & & & & & & & + \\
\hline \multirow[t]{2}{*}{10} & Case & & + & & + & & & & + & + & & & + & & & + & & & + & + & & & & + & + \\
\hline & Control & & & + & & & + & & + & + & + & & & + & & & & & + & & & & & & + \\
\hline \multirow[t]{2}{*}{11} & Case & + & + & + & & & & & & + & + & + & & + & & & & & + & & & + & & & + \\
\hline & Control & & + & + & & & & & & & + & & & & & & & & & & & & & & + \\
\hline \multirow[t]{2}{*}{12} & Case & & + & & & & & & + & & + & & + & & & & & & + & & & & & & + \\
\hline & Control & & & & & & & & & & + & & + & & & & & & & + & & & & & + \\
\hline \multirow[t]{2}{*}{13} & Case & & & & + & + & & + & + & & & & & + & & & & & & & & & & & + \\
\hline & Control & & & & + & & & & + & & & & & + & & & & & & + & & & & & + \\
\hline & al farms (n) & 1 & 6 & 3 & 4 & 2 & 4 & 4 & 6 & 3 & 5 & 1 & 6 & 7 & 2 & 2 & 2 & 1 & 5 & 8 & 3 & 5 & 1 & 2 & 11 \\
\hline
\end{tabular}

+: Present; un: Untypable. 


\subsection{S. suis Serotypes Recovered from Systemic Sites}

The S. suis serotypes recovered from systemic sites of confirmed cases are shown in Table 3 . The most commonly detected serotypes found in systemic sites at the pig level were $9(n=9),(2,1 / 2)(n=7)$, and untypable isolates $(n=7)$. The most commonly detected serotypes found in systemic sites at the farm level included 29 on three farms, $(2,1 / 2)$ and nine each on two farms, as well as untypable isolates on four farms.

Table 3. Pattern positivity for different serotypes found in systemic sites (blood, meninges, spleen) of 28 confirmed cases of Streptococcus suis infections at the pig and farm level.

\begin{tabular}{cccccccccccccccccc}
\hline $\begin{array}{c}\text { Pattern } \\
\text { id\# }\end{array}$ & $\begin{array}{c}\text { Pigs } \\
\text { (n) }\end{array}$ & $\begin{array}{c}\text { Farms } \\
\text { (n) }\end{array}$ & $\begin{array}{c}\text { Serotype } \\
\text { (n) }\end{array}$ & un & $\mathbf{1 , 1 4}$ & $\mathbf{2 , 1 / 2}$ & $\mathbf{3}$ & $\mathbf{4}$ & $\mathbf{7}$ & $\mathbf{8}$ & $\mathbf{9}$ & $\mathbf{1 5}$ & $\mathbf{1 8}$ & $\mathbf{2 8}$ & $\mathbf{2 9}$ & $\mathbf{3 1}$ \\
\hline 1 & 1 & 1 & 1 & - & - & - & - & - & - & - & - & - & - & - & - & + \\
2 & 1 & 1 & 1 & - & - & - & - & - & - & - & - & - & + & - & - & - \\
3 & 6 & 1 & 1 & - & - & - & - & - & - & - & + & - & - & - & - & - \\
4 & 1 & 1 & 3 & - & - & - & - & - & - & - & + & - & - & + & + & - \\
5 & 1 & 1 & 2 & - & - & - & - & - & - & - & + & + & - & - & - & - \\
6 & 1 & 1 & 1 & - & - & - & - & - & + & - & - & - & - & - & - & - \\
7 & 2 & 1 & 1 & - & - & - & - & + & - & - & - & - & - & - & - & - \\
8 & 1 & 1 & 1 & - & - & - & + & - & - & - & - & - & - & - & - & - \\
9 & 5 & 2 & 1 & - & - & + & - & - & - & - & - & - & - & - & - & - \\
10 & 1 & 1 & 2 & - & - & + & - & - & - & - & - & - & + & - & - & - \\
11 & 1 & 1 & 3 & - & - & + & - & - & - & - & - & - & + & - & + & - \\
12 & 1 & 1 & 1 & - & + & - & - & - & - & - & - & - & - & - & - & - \\
13 & 2 & 1 & 1 & + & - & - & - & - & - & - & - & - & - & - & - & - \\
14 & 1 & 1 & 2 & + & - & - & - & - & - & - & - & - & - & - & + & - \\
15 & 1 & 1 & 2 & + & - & - & - & - & - & - & + & - & - & - & - & - \\
16 & 1 & 1 & 2 & + & - & - & - & - & - & + & - & - & - & - & - & - \\
17 & 1 & 1 & 2 & + & + & - & - & - & - & - & - & - & - & - & - & - \\
\hline Pigs (n) & 28 & n/a & & 6 & 2 & 7 & 1 & 2 & 2 & 1 & 9 & 1 & 3 & 1 & 3 & 1 \\
Farms (n) & n/a & 8 & & 4 & 1 & 2 & 1 & 1 & 2 & 1 & 2 & 1 & 1 & 1 & 2 & 1 \\
\hline
\end{tabular}

+: Positive; -: Negative; un: Untypable.

Seventeen confirmed cases (61\%) were identified with a single serotype in systemic sites, as well as nine cases (32\%) with two serotypes, and two cases $(7 \%)$ with three serotypes. The most common pattern of positivity for serotypes was positivity for serotype 9 only $(n=6)$, followed by positivity for serotype $(2,1 / 2)$ only $(n=5)$ (Table 3). Furthermore, the most common pattern of positivity seen on farms in confirmed cases was detection of serotype $(2,1 / 2)(n=2$ farms) (Table 3$)$.

\subsection{Serotypes from Systemic Sites (Blood, Meninges and Spleen) Compared to Serotypes in Upper Respiratory} Sites (Tonsils and Nasal Cavity) of Case Pigs

Streptococcus suis serotypes were not recovered from upper respiratory sites in seven confirmed ( $25 \%)$ cases. Similarly, a single serotype was also identified in seven confirmed cases $(25 \%)$, as well as two serotypes in $10(35.71 \%)$ confirmed cases, three serotypes $(10.71 \%)$ in three confirmed cases, and five serotypes $(3.57 \%)$ in one confirmed case. The same serotypes were recovered from systemic and upper respiratory sites in six $(21.4 \%)$ confirmed cases. This included $(2,1 / 2)(n=2), 9(n=2), 18(n=1)$, and $31(\mathrm{n}=1)$.

When univariable logistic regression was performed, there was an interest in how untypable isolates and serotypes 9 and $(2,1 / 2)$ compared between systemic and upper respiratory sites in confirmed cases (Table 4). Untypable isolates $(n=5)$ were frequently recovered in pigs in both sites, although there was no association between their detection in systemic and upper respiratory sites. Conversely, the presence of serotype $9(\mathrm{OR}=5.6, p=0.03)$ in upper respiratory sites were positively associated with the detection of the same serotype in the systemic sites of the same pig, while a similar positive trend was seen for serotype $(2,1 / 2)(\mathrm{OR}=7.2, p=0.07)$. However, it should be noted that 
isolation of serotypes found in both systemic and upper respiratory sites was low. This was also reflected in the low estimates of population attributable fraction for both serotypes (Table 4).

Table 4. Serotypes found in systemic (blood, meninges, and spleen) and upper respiratory (tonsil and nasal cavity) sites of 28 confirmed $S$. suis cases.

\begin{tabular}{|c|c|c|c|c|c|c|c|c|}
\hline \multirow{2}{*}{ Serotype } & \multicolumn{2}{|c|}{ Upper Resp $^{a}(+)$} & \multicolumn{2}{|c|}{ Upper Resp (-) } & \multirow{2}{*}{$\mathrm{OR}^{\mathrm{c}}$} & \multirow{2}{*}{ PAF $^{d}$} & \multirow{2}{*}{$\mathrm{CI}^{\mathrm{e}}$} & \multirow{2}{*}{$P^{f}$} \\
\hline & Sys $^{b}(+)$ & Sys (-) & Sys (+) & Sys (-) & & & & \\
\hline 1,14 & 0 & 0 & 2 & 26 & $\mathrm{~Np} g$ & $\mathrm{np}$ & $\mathrm{np}$ & $\mathrm{np}$ \\
\hline $2,1 / 2$ & 2 & 1 & 5 & 20 & 7.24 & 0.20 & $0.319,494.7$ & 0.07 \\
\hline 3 & 0 & 1 & 1 & 26 & $\mathrm{np}$ & $\mathrm{np}$ & np & $\mathrm{np}$ \\
\hline 4 & 1 & 0 & 2 & 25 & - & - & - & 0.78 \\
\hline 7 & 0 & 0 & 2 & 26 & $\mathrm{np}$ & $\mathrm{np}$ & $\mathrm{np}$ & $\mathrm{np}$ \\
\hline 8 & 0 & 0 & 1 & 27 & np & np & np & np \\
\hline 9 & 2 & 0 & 7 & 19 & 5.59 & 0.16 & $0.4151,+$ inf & 0.03 \\
\hline 10 & 0 & 1 & 0 & 27 & $\mathrm{np}$ & $\mathrm{np}$ & $\mathrm{np}$ & np \\
\hline 11 & 0 & 2 & 0 & 26 & np & np & np & np \\
\hline 15 & 1 & 2 & 0 & 25 & $\mathrm{np}$ & np & $\mathrm{np}$ & $\mathrm{np}$ \\
\hline 16 & 0 & 0 & 4 & 24 & np & np & np & np \\
\hline 18 & 1 & 1 & 1 & 25 & np & np & np & np \\
\hline 28 & 0 & 0 & 1 & 27 & np & np & np & - \\
\hline 29 & 0 & 1 & 3 & 24 & - & - & - & 0.72 \\
\hline 31 & 1 & 2 & 0 & 25 & $\mathrm{np}$ & $\mathrm{np}$ & $\mathrm{np}$ & $\mathrm{np}$ \\
\hline $\mathrm{Un}^{\mathrm{h}}$ & 4 & 12 & 1 & 11 & - & - & - & 0.25 \\
\hline
\end{tabular}

${ }^{a}$ Upper resp: upper respiratory sites; ${ }^{b}$ sys: Systemic sites; +: Positive; -: Negative; OR ${ }^{c}$ : Odds ratio; PAF ${ }^{d}$ : Population attributable fraction; $\mathrm{CI}^{\mathrm{e}}$ : Confidence interval; $\mathrm{p}^{\mathrm{f}}$ : $p$-value. Note that confidence intervals and $p$-values were calculated using two different methods. $\mathrm{Np}^{\mathrm{g}}=$ Not performed; $\mathrm{un}^{\mathrm{h}}$ : Untypable.

\subsection{S. suis Serotypes in Upper Respiratory Sites (Tonsils and Nasal Cavity) of Case and Control Pigs}

Together, 40 different serotype patterns were found in upper respiratory sites of 59 case and 59 matched control animals. The most frequent patterns included only untypable isolates in case $(\mathrm{n}=12)$ and control $(\mathrm{n}=18)$ animals, along with no $S$. suis recovered in case $(\mathrm{n}=13)$ and control $(\mathrm{n}=15)$ animals. In the subset of animals used for this analysis, zero to four serotypes were detected in upper respiratory sites of case and control animals. The most frequent serotypes in case animals included $(2,1 / 2)(n=8), 15(n=6), 9(n=5), 29(n=5), 30(n=5)$, and $16(n=4)$, along with 21 isolates which remained untypable. The most frequent serotypes in control animals included $29(n=6), 7(n=3), 16$ $(n=4), 17(n=3)$, and $18(n=3)$, along with 29 isolates which remained untypable.

Seven matched case-control pairs $(11.8 \%)$ shared at least one of the same serotypes in upper respiratory sites and can be seen in Table 5. These included serotype $(2,1 / 2)$ from two farms $(n=3)$, serotype 18 from one farm $(n=2)$, serotype 30 from one farm $(n=1)$, and serotype 16 from one farm $(\mathrm{n}=1)$. Untypable isolates were also present on eight farms. Univariable conditional logistic regressions showed there was no statistical evidence to suggest that the presence of a serotype in controls was associated with its presence in cases (Table 5). 
Table 5. Streptococcus suis serotypes found in upper respiratory (tonsil and nasal cavity) sites of 59 clinically ill and 59 healthy pigs.

\begin{tabular}{|c|c|c|c|c|}
\hline Serotype & Clinically Ill Pigs (n) & Healthy Pigs (n) & (n) Matched Pairs ((n) on Farms) & $\mathbf{P}^{\mathrm{c}}$ \\
\hline $2,1 / 2$ & 7 & 4 & 3 (2 farms) & 0.124 \\
\hline 3 & 3 & 1 & $1(1$ farm $)$ & $\mathrm{Np}^{\mathrm{d}}$ \\
\hline 4 & 1 & 1 & - & 1 \\
\hline 7 & 1 & 3 & - & 0.21 \\
\hline 9 & 5 & 2 & - & 0.27 \\
\hline 10 & 2 & 1 & - & 0.57 \\
\hline 11 & 3 & 2 & - & 0.66 \\
\hline 12 & 1 & 0 & - & 1 \\
\hline 15 & 6 & 0 & - & $\mathrm{np}$ \\
\hline 16 & 4 & 4 & 1 (1 farm) & 1 \\
\hline 17 & 0 & 3 & - & $\mathrm{np}$ \\
\hline 18 & 3 & 3 & 2 (1 farm) & 1 \\
\hline 21 & 2 & 0 & - & $\mathrm{np}$ \\
\hline 28 & 2 & 2 & - & 1 \\
\hline 29 & 5 & 6 & - & 0.566 \\
\hline 30 & 5 & 0 & - & $\mathrm{np}$ \\
\hline 31 & 2 & 1 & - & 0.341 \\
\hline 33 & 1 & 0 & - & $\mathrm{np}$ \\
\hline $\mathrm{Un}^{\mathrm{e}}$ & 21 & 29 & 15 (8 farms) & 0.301 \\
\hline
\end{tabular}

\section{Discussion}

Diseases caused by Streptococcus suis are some of the most common bacterial diseases in the nursery stage of swine production. With a high frequency of healthy pigs carrying S. suis, there is a knowledge gap with respect to why some pigs become sick while others remain unaffected.

According to previous studies, the major serotypes of diseased pigs in North America have been 2,3 , and $1 / 2[8,9]$. There are some differences between these previously reported data and the present study. However, serotype $(2,1 / 2)$ was the most frequently isolated serotype found in clinical cases. Serotype 3 appeared in both case and control animals but was not found as frequently as the other serotypes. Interestingly, serotype 9 , which, in previous studies has rarely been isolated from diseased pigs in Canada, appeared as frequently as serotype $(2,1 / 2)$ and its emergence has been shown in recent years [10].

In the present study, three different types of confirmed cases could be identified from descriptive statistics of positivity to different serotypes. First, there were $S$. suis cases which identified primarily with serotypes 9 and $(2,1 / 2)$ alone or in combination with other serotypes. The identification of clusters of cases within individual farms would be most consistent with either the clonal spread of one or more linkages of the same serotype among pigs, or more generally to the same source of infection $[2,11]$. Consistent with our research, a recent study in Germany also found systemic infections associated with serotype $(2,1 / 2)$ and serotype 9 [12]. Second, there was a subset of confirmed cases that were all detected with untypable isolates, alone or in combination with other serotypes. More discriminatory molecular typing is required in an attempt to understand the incidence of such confirmed cases. In the previous literature, untypable isolates were hypothesized to be defective mutants of strains of known serotypes or represent novel serotypes [8]. Last, there were confirmed cases that were detected with only one serotype, other than serotypes 9 or $(2,1 / 2)$. Such cases were observed in low frequency, with a maximum of two detections of serotype 3 on one farm. The low number of investigated clinical cases on some farms could have influenced this result and our interpretation. However, such detection is most consistent with existence of sporadic cases associated with different serotypes. These cases may be due to coinfections that have enabled the bacterium to reach systemic sites of these pigs [13]. 
Previous studies have also shown that $S$. suis serotypes recovered from systemic sites were generally different compared to those found in upper respiratory sites [14,15]. In the present study, only $21 \%$ of confirmed cases had serotypes in systemic sites that were the same to those in upper respiratory sites of the same pigs. When individual serotypes were evaluated, positive association between detection in upper respiratory sites and detection in systemic sites could be found only for serotypes 9 and a trend for serotype $(2,1 / 2)$. However, even for these two serotypes, only a small fraction of cases due to a specific serotype could be linked with detection of the same serotype in upper respiratory sites. Since multiple serotypes can colonize pigs, it is possible that our sampling and testing strategy resulted in low sensitivity of detecting a specific serotype in upper respiratory sites. However, this result also suggests that, when evaluated using a typical sampling and testing protocol, serotypes found in upper respiratory sites are not necessarily the same serotypes found in systemic sites causing pigs to become sick.

Further, there was no association between the presence of a serotype in upper respiratory sites of case and control animals. This suggests that we could not identify evidence that pen-mates serve as the source of colonization for cases, or that there is a common source of colonization for the majority of serotypes. Nonetheless, a combination of study design, small sample size and, possibly, low sensitivity of detecting a specific serotype in upper respiratory sites could also have negatively affected our ability to detect such associations.

This study has several limitations. It is possible that the clinically ill pigs were treated with an antibiotic prior to sampling, and as a result, some bacteria could have been eliminated from the blood and/or meninges and would therefore only be present in upper respiratory sites [16]. For the purpose of this study, we considered a combination of results from tonsillar and nasal swabs to be representative of the upper respiratory tract. It should be noted that not all sections of the upper respiratory tract could be sampled. Multiple serotypes were also found in systemic sites and could be suggestive of infection with more than one serotype and/or contamination. Consistent with previous observations reported, the use of the two-step multiplex PCR was also not able to distinguish between serotypes 1 and 14 and 2 and 1/2 [17,18]. Since we used PCR typing, we could have used the term capsular types as a surrogate for serotypes, but we decided to use serotypes in order to be consistent with the literature. Additionally, only one healthy animal was tested for each sick animal. Therefore, the diversity of serotypes at the farm level may have been underestimated. Last, it is also possible that issues with processing of samples such as transporting, storing, and culturing could have resulted in false negative results. Nevertheless, as many samples were obtained from both sick and healthy pigs, a snapshot of the current situation of $S$. suis in Ontario nurseries can be seen.

\section{Conclusions}

In conclusion, most commonly detected serotypes in systemic infections in S. suis diseased pigs in this study were serotypes $(2,1 / 2)$ and 9 , followed by frequent isolation of PCR untypable isolates from systemic sites. The colonization of upper respiratory sites with serotype 9 was positively associated with detection of these serotypes in systemic sites while a trend was seen for serotype $(2,1 / 2)$. However, only a small fraction of detection in systemic sites could be linked with the detection of the same serotype in upper respiratory sites (up to $20 \%$, as indicated by the estimate of the population attribution fraction), making this result of limited practical use. Last, no link between the colonization of upper respiratory sites of case and control animals could be detected. Further research on the S. suis whole genome needs to be conducted to distinguish molecular differences of isolates. The high detection frequency of untypable isolates warrants further investigation. 


\section{Methods}

\subsection{Study Design and Sampling}

A sample size calculation was based on visual inspection of power curves that were generated for a matched case-control study design using a power of $80 \%$, confidence of $95 \%$, and a ratio of cases and controls of 1 . The prevalence of exposure in the control group was assumed to vary between a minimum of 0.12 and a maximum of 0.3 . Under such assumptions, the number of cases and controls required to detect an odds ratio of 3 varied between 58 (control group exposure of 0.12 ) and 97 (control group exposure of 0.3). The required sample size was 31 cases if the magnitude of the odds ratio was 4 . For field sampling, we targeted a minimum of 60 clinical cases and matched controls due to expected between farm variability and anticipated exclusion of clinical cases due to inadequate detection of $S$. suis in the sampling sites.

Owners of 12 farms located in Ontario, Canada, expressed interest in participating in this study because nursery pigs raised on these farms were experiencing typical clinical signs of $S$. suis infection.

Pigs showing clinical signs of $S$. suis infection (cases) were selected on each farm. For a pig to be considered a clinical case, they needed to exhibit at least one of the following clinical signs: Ataxia, incoordination, tremor/shaking, paddling, opisthotonos, paralysis, convulsions, and/or nystagmus. Each case was matched with an equal number of healthy pigs (controls) on each herd based on the time of visit and pen. For a pig to be considered a control, they needed to exhibit healthy and normal behavior, described as the absence of any of the above listed clinical signs.

Each case and control animal had one nostril, its tonsils, and its rectum swabbed, and blood sampled. In addition, cases were sedated with an intramuscular injection of a combination of ketamine $(50 \mathrm{mg} / \mathrm{mL})$, xylazine $(10 \mathrm{mg} / \mathrm{mL})$, and butorphanol $(1 \mathrm{mg} / \mathrm{mL})$ with a dosage of $0.2 \mathrm{~mL} / \mathrm{kg}$ body weight and then euthanized with a 3-mL intracardiac injection of pentobarbital sodium $(240 \mathrm{mg} / \mathrm{mL})$. The skull was opened to collect meningeal swabs, and tissue samples were collected from the spleen. Samples were placed in a cooler after collection on the farm and brought to the laboratory located at the Centre for Public Health and Zoonoses (CPHAZ) at the University of Guelph for analysis. Samples were in the cooler for between $20 \mathrm{~min}$ and $2 \mathrm{~h}$ depending on the distance travelled to the farm.

\subsection{S. suis Isolation and Identification}

\subsubsection{S. suis Culturing}

Tissue samples were placed in a paper boat and cut with a sterilized scalpel. Spleen samples, blood, and swabs (meninges, tonsil, nasal cavity, rectal) were plated on phenylethyl alcohol (PEA) agar and incubated at $35{ }^{\circ} \mathrm{C}$ with $5 \% \mathrm{CO}_{2}$ for $48 \mathrm{~h}$. One to four suspected $\mathrm{S}$. suis colonies were selected per sample and plated on PEA agar and incubated at $35^{\circ} \mathrm{C}$ with $5 \% \mathrm{CO}_{2}$ for $48 \mathrm{~h}$.

\subsubsection{DNA Extraction}

Multiple suspected colonies were selected from PEA and DNA was extracted from isolates using InstaGene Matrix (Bio-Rad, Hercules, CA, USA) according to the manufacturer's instructions. DNA samples were stored at $-20^{\circ} \mathrm{C}$.

\subsubsection{S. suis Identification}

To identify S. suis, DNA extracted from all selected suspected colonies were tested for the presence of the glutamate dehydrogenase $(g d h)$ gene [19] and the recombination protein $\mathrm{N}(\mathrm{recN})$ gene by PCR [20]. The isolates were confirmed as S. suis if both genes were present. The confirmed S. suis isolates were then stored in CryoStor microbiology culture reservation vials according to the manufacturer's instructions (BioLife Solutions, Bothell, WA, USA) for further identification. 


\subsubsection{Serotyping}

The S. suis isolates were analyzed using a two-step multiplex PCR method as described previously [18]. Capsular polysaccharide types determined by the PCR were examined by electrophoresis on a 1.5\% agarose gel and visualized with RedSafe Nucleic Acid Staining Solution (FroggaBio, Toronto, ON, Canada).

\subsubsection{Categorization of Cases}

Case pigs were divided into two major groups, including 28 confirmed cases and 32 probable cases. A pig was classified as a "confirmed case" if the pig displayed clinical signs at the time of visit and had the presence of $S$. suis recovered in systemic sites, including the blood, meninges, and/or spleen. A pig was classified as a "probable case" if the pig displayed clinical signs at the time of visit and had the presence of $S$. suis only recovered in any of the other locations sampled (tonsils, nasal cavity, or rectum). Four cases did not have $S$. suis detected from any site.

\subsection{Statistical Analysis}

Data were entered into a spreadsheet (Microsoft Excel 2016), cleaned, and then imported into Stata 15 (StataCorp, College Station, Texas, TX, USA) for further processing, descriptive statistics, and visualization. Descriptive statistics were conducted at the isolate level, pig level, and farm level. The isolate-level dataset was aggregated to a pig-level dataset, with individual variables representing the presence of $S$. suis to specific sampling sites for specific serotypes in individual pigs. Additional summary statistics were used to describe the detection of serotypes in upper respiratory sites and from rectal swabs in all available clinical case and control animals.

Objective 1. Twenty-eight confirmed cases were used to address Objective 1. Patterns of positivity were assessed by aggregating the number of confirmed cases detected with a specific serotype and with a combination of specific serotypes.

Objective 2. To investigate systemic and upper respiratory sites, the same 28 confirmed cases were used. A series of univariable exact logistic regression models was applied to investigate associations between the presence of a specific serotype in upper respiratory sites as a risk factor for detection of the same serotype in systemic sites of the same confirmed case. Analysis was performed only for serotypes that had at least three cases identified, and estimates were reported if $p<0.10$. Population attributable fraction was determined using contingency tables.

Objective 3. To investigate the upper respiratory sites, only matched pairs that had sampling from both tonsil and nasal swabs were included for this objective. This resulted in a total of 59 of 64 clinically ill pigs and 59 of 64 individually matched healthy pigs that fit these criteria. A series of univariable conditional logistic regression models were used to investigate the association between the presence of a specific serotype in upper respiratory sites of clinically ill and healthy animals. Estimates were reported if $p<0.10$.

Author Contributions: Conceptualization, L.C.D., A.F., R.F. and Z.P.; Data curation, L.C.D. and Z.P.; Formal analysis, L.C.D. and Z.P.; Investigation, L.C.D.; Methodology, L.C.D., A.F., R.F. and E.A.; Project administration, A.F., R.F. and Z.P.; Resources, A.F., R.F. and Z.P.; Supervision, A.F., R.F. and Z.P.; Visualization, L.C.D. and Z.P.; Writing—original draft, L.C.D.; Writing—review \& editing, L.C.D., A.F., R.F., M.G. and Z.P. All authors have read and agreed to the published version of the manuscript.

Funding: Funding for this project was provided by the Canada First Research Excellence Fund by the Food from Thought research program at the University of Guelph, Ontario, Canada.

Acknowledgments: We are grateful for the assistance of swine practitioners from South West Ontario Veterinary Services and Demeter Services Vétérinaires Inc. as well as the Ontario pork producers for volunteering to be a part of the study.

Conflicts of Interest: The authors declare that they have no conflicts of interests that could inappropriately influence this work. 
Ethics Approval and Consent to Participate: This research project was conducted with approval from the University of Guelph Animal Care Committee and followed the guidelines of the Canadian Council on Animal Care. Consent was also obtained from participating farm owners and followed appropriate guidelines from the University of Guelph. (AUP number: 3820; REB number: 16-12-244)

\section{Abbreviations}

DNA: deoxyribonucleic acid; $g d h$ : glutamate dehydrogenase; PCR: polymerase chain reaction; PEA: phenylethyl alcohol agar; recN: recombination protein N; S. suis: Streptococcus suis.

\section{References}

1. Gottschalk, M.; Segura, M. The pathogenesis of the meningitis caused by Streptococcus suis: The unresolved questions. Vet. Microbiol. 2000, 76, 259-272. [CrossRef]

2. Cloutier, G.; D'allaire, S.; Martinez, G.; Surprenant, C.; Lacouture, S.; Gottschalk, M. Epidemiology of Streptococcus suis serotype 5 infection in a pig herd with and without clinical disease. Vet. Microbiol. 2003, 97, 135-151. [CrossRef] [PubMed]

3. Lun, Z.R.; Wang, Q.P.; Chen, X.G.; Li, A.X.; Zhu, X.Q. Streptococcus suis: An emerging zoonotic pathogen. Lancet Infect. Dis. 2007, 7, 201-209. [CrossRef]

4. Goyette-Desjardins, G.; Auger, J.P.; Xu, J.; Segura, M.; Gottschalk, M. Streptococcus suis, an important pig pathogen and emerging zoonotic agent-An update on the worldwide distribution based on serotyping and sequence typing. Emerg. Microbes Infect. 2014, 3, 45. [CrossRef] [PubMed]

5. Vela, A.I.; Goyache, J.; Tarradas, C.; Luque, I.; Mateos, A.; Moreno, M.A.; Borge, C.; Perea, J.A.; Dominguez, L.; Fernandez-Garayzabal, J.F. Analysis of genetic diversity of Streptococcus suis clinical isolates from pigs in Spain by pulsed-field gel electrophoresis. J. Clin. Microbiol. 2003, 41, 2498-2502. [CrossRef] [PubMed]

6. Gottschalk, M. Streptococcosis. In Diseases of Swine; Zimmerman, J.J., Karriker, L.A., Rameirez, A., Schwartz, K.J., Stevenson, G.W., Eds.; Wiley-Blackwell Publishing: Ames, IA, USA, 2019; pp. 934-950.

7. Higgins, R.; Gottschalk, M.; Beaudoin, M.; Rawluk, S.A. Distribution of Streptococcus suis capsular types in Quebec and western Canada. Can. Vet. J. 1992, 33, 27. [PubMed]

8. Gottschalk, M.; Lacouture, S.; Bonifait, L.; Roy, D.; Fittipaldi, N.; Grenier, D. Characterization of Streptococcus suis isolates recovered between 2008 and 2011 from diseased pigs in Quebec, Canada. Vet. Microbiol. 2013, 162, 819-825. [CrossRef] [PubMed]

9. Haas, B.; Grenier, D. Understanding the virulence of Streptococcus suis: A veterinary, medical, and economic challenge. Infect. Dis. 2018, 48, 159-166. [CrossRef] [PubMed]

10. Zheng, H.; Du, P.; Qiu, X.; Kerdsin, A.; Roy, D.; Bai, X.; Gottschalk, M. Genomic comparisons of Streptococcus suis serotype 9 strains recovered from diseased pigs in Spain and Canada. Vet. Res. 2018, 49, 1. [CrossRef] [PubMed]

11. Dekker, N.; Bouma, A.; Daemen, I.; Vernooij, H.; van Leengoed, L.; Wagenaar, J.; Stegeman, A. Effect of simultaneous exposure of pigs to Streptococcus suis serotypes 2 and 9 on their colonization and transmission, and on mortality. Pathogens 2018, 6, 46. [CrossRef] [PubMed]

12. Prüfer, T.L.; Rohde, J.; Verspohl, J.; Rohde, M.; de Greeff, A.; Willenborg, J.; Valentin-Weigand, P. Molecular typing of Streptococcus suis strains isolated from diseased and healthy pigs between 1996-2016. PLoS ONE 2019, 14, e0210801. [CrossRef] [PubMed]

13. Feng, W.H.; Laster, S.M.; Tompkins, M.; Brown, T.; Xu, J.S.; Altier, C.; McCaw, M.B. In utero infection by porcine reproductive and respiratory syndrome virus is sufficient to increase susceptibility of piglets to challenge by Streptococcus suis type II. J. Virol. 2001, 75, 4889-4895. [CrossRef] [PubMed]

14. Oliveira, S.; Batista, L.; Torremorell, M.; Pijoan, C. Experimental colonization of piglets and gilts with systemic strains of Haemophilus parasuis and Streptococcus suis to prevent disease. Can. J. Vet. Res. 2001, 65, 161. [PubMed]

15. Torremorell, M.; Calsamiglia, M.; Pijoan, C. Colonization of suckling pigs by Streptococcus suis with particular reference to systemic serotype 2 strains. Can. J. Vet. Res. 1998, 62, 21-26. [PubMed]

16. Fittipaldi, N.; Segura, M.; Grenier, D.; Gottschalk, M. Virulence factors involved in the pathogenesis of the infection caused by the swine pathogen and zoonotic agent Streptococcus suis. Future Microbiol. 2012, 7, 259-279. [CrossRef] [PubMed] 
17. Liu, Z.; Zheng, H.; Gottschalk, M.; Bai, X.; Lan, R.; Ji, S.; Xu, J. Development of multiplex PCR assays for the identification of the 33 serotypes of Streptococcus suis. PLoS ONE 2013, 8, 72070. [CrossRef] [PubMed]

18. Okura, M.; Lachance, C.; Osaki, M.; Sekizaki, T.; Maruyama, F.; Nozawa, T.; Takamatsu, D. Development of a two-step multiplex PCR assay for typing of capsular polysaccharide synthesis gene clusters of Streptococcus suis. J. Clin. Microbiol. 2014, 52, 1714-1719. [CrossRef] [PubMed]

19. Okwumabua, O.; O'Connor, M.; Shull, E. A polymerase chain reaction (PCR) assay specific for Streptococcus suis based on the gene encoding the glutamate dehydrogenase. FEMS Microbiol. Lett. 2013, 218, 79-84. [CrossRef] [PubMed]

20. Ishida, S.; le Tien, H.T.; Osawa, R.; Tohya, M.; Nomoto, R.; Kawamura, Y.; Takahashi, T.; Kikuchi, N.; Kikuchi, K.; Sekizaki, T. Development of an appropriate PCR system for the reclassification of Streptococcus suis. J. Microbiol. Methods 2014, 107, 66-70. [CrossRef] [PubMed]

(C) 2020 by the authors. Licensee MDPI, Basel, Switzerland. This article is an open access article distributed under the terms and conditions of the Creative Commons Attribution (CC BY) license (http://creativecommons.org/licenses/by/4.0/). 